\title{
Intermédialités
}

Histoire et théorie des arts, des lettres et des techniques

Intermediality

History and Theory of the Arts, Literature and Technologies

\section{Paysages de la ville électronique}

\section{Lucio Altarelli}

Numéro 14, automne 2009

bâtir

build

URI : https://id.erudit.org/iderudit/044410ar

DOI : https://doi.org/10.7202/044410ar

Aller au sommaire du numéro

Éditeur(s)

Revue Intermédialités (Presses de l’Université de Montréal)

ISSN

1705-8546 (imprimé)

1920-3136 (numérique)

Découvrir la revue

Citer cet article

Altarelli, L. (2009). Paysages de la ville électronique. Intermédialités /

Intermediality, (14), 67-84. https://doi.org/10.7202/044410ar
Résumé de l'article

Le passage du Moderne à la surmodernité de la révolution informatique sanctionne un déplacement graduel des catégories du visible, de la permanence et de la prévision vers celles de la transparence, de la légèreté et de la virtualité produites dans un contexte de fluidité provisoire. Le passage de l'éden électrique du Moderne à l'éden électronique du contemporain trace le contour de quatre thèmes qui configurent à leur tour autant d'images de la ville : la ville opalescente, la ville médiale, la ville évanescente et la ville relationnelle. Dans l'ensemble, ces représentations de la ville tendent à une légèreté commune. 


\title{
Paysages de la ville électronique
}

\author{
Lucio Altarelli
}

e thème de la lumière artificielle et de ses incidences urbaines constitue un

fil conducteur qui lie le Moderne au contemporain, tout en présentant des analogies et des différences. Dans le Moderne, la lumière est liée à l'effet Edison: l'invention de la lampe à incandescence qui fait irruption à l'intérieur de la scène urbaine donne lieu à l'urbanisme de la nuit. À l'inverse, dans le contemporain, la lumière est médiatisée par le paradigme électronique et par la culture digitale. La découverte d'Edison constitue, dans le Moderne, l'incipit d'un merveilleux urbain qui se déploie à l'échelle planétaire, investissant autant les centres-villes du Nouveau Monde que les cités du vieux continent. Au début du siècle dernier, les pyroscaphes maritimes favorisent les échanges entre l'Amérique et l'Europe. C'est à cette époque que l'éden électronique fleurit au sein du nouveau continent, dans les premiers parcs d'amusement, dans les lumières de Dreamland et de Coney Island, dans l'illusionnisme des Panoramas, dans les panneaux lumineux de Broadway et dans les flèches des gratte-ciel, véritables phares terrestres.

Au cours des années 1920 et 1930, l'Europe répond à ces sollicitations par la réalisation de bâtiments qui conjuguent les suggestions du verre avec les enseignes de la publicité, donnant lieu par là à un véritable langage architectonique: celui de la Lichtarchitektur et de la Reklame Architektur. Le sublime urbain de ces architectures exalte le rôle de la lumière artificielle et réduit à néant celui joué auparavant par la lumière naturelle. Il dépasse l'idée lecorbusienne de l'architecture comme jeu savant, correct et magnifique des volumes assemblés dans la lumière. En faisant explicitement appel aux «cinq points» de Le Corbusier, André Lurçat en corrige le tir par l'introduction de la lumière artificielle. Dans Architecture, datant de 1929, trois ans après Le Corbusier, il indique les «nouveaux éléments» de cette architecture: "pilotis», «terrasses», «fenêtres», «couleur» et, notamment, «lumière artificielle ${ }^{1}$ ».

1. André Lurçat, Architecture [1929], dans Marco De Michelis, Vittorio Magnano Lampugnani, M. Pogačnik et R. Scheider (dir.), Espressionismo e nuova oggettività. La 
L’Éden électrique trouve une nouvelle déclinaison, entre langage pop et dérives kitsch, dans le Las Vegas des années 1970. Cette déclinaison est analysée en profondeur par Venturi, Scott Brown et Izenour dans le célèbre pamphlet Learning from Las Vegas (1972; texte traduit en italien au cours de la seconde moitié des années 1980). Édifié comme un prodigieux collage de monuments du passé, tel un patchwork enchevêtré surgissant au milieu d'une jubilation de pyramides égyptiennes, arcs de triomphe et palais ducaux, Las Vegas apparaît à Rem Koolhaas sous le jour d'une «ville staliniste »; en effet, par ses bâtiments colossaux, elle reproduit la monumentalité des anciennes architectures du régime en dépit de son langage éphémère et vacancier ${ }^{2}$. Les paysages électriques qui, à partir de la Reklame Architektur des années 1930, se déploient jusqu’au monumentalisme lumineux de Las Vegas, représentent les daguerréotypes jaunis d'une modernité qui, arrivée au bout de sa trajectoire, passe le relais aux nouveaux districts du numérique, traçant la cartographie d'un nouveau sublime urbain qui opère au nom et pour le compte du numérique et du virtuel.

Aujourd'hui, les langages de l'architecture, tout comme les installations de l'art et de la culture, s'avèrent toujours plus impliqués et conditionnés par les sollicitations qui proviennent des paysages du numérique, où convergent avec force détails les nouveaux médias, l'informatique et l'art électronique qui, de concert avec le délit parfait mis en œuvre par les processus de déréalisation et de délocalisation, donnent lieu à cette perte de matière et de consistance qui marque le passage du Moderne au contemporain. Comme on sait, le passage du Moderne à la surmodernité de la révolution informatique provoque un glissement graduel des catégories du visible, de la permanence et de la prévision à celles de la transparence, de la légèreté et de la virtualité, dans un contexte de fluidité provisoire. Avec le passage de l'éden électrique du Moderne à l'éden électronique du contemporain, quatre paysages thématiques commencent donc à prendre forme, donnant lieu à autant de paysages urbains.

\section{LA VILLE OPALESCENTE}

La ville opalescente est la ville des reflets et des fondus enchaînés qui se découpent à même les devantures translucides et transparentes des magasins, des bureaux, des mégastores et des galeries d'art, en un jeu de chatoiements et

nuova architettura europea degli anni venti, Milan, La Triennale di Milano, Electa, 1994, p. 102.

2. François Chaslin, Deux conversations avec Rem Koolhaas, et cætera, Paris, Sens \& Tonka, 2001. 
de réverbérations qui amplifie à l'infini le miroir magique d'Alice: comme dans le récit de Lewis Carroll, nous pouvons fixer les images générées par la surface réfléchissante ou bien traverser le miroir, pénétrer dans son univers complémentaire, où tout est à l'envers et dans lequel il faut «marcher dans la direction opposée » à celle où l'on désire aller. Au milieu de cette accumulation frénétique de lumières et de couleurs, de reflets urbains et d'introspections, de public et de privé, d'éléments statiques et d'éléments dynamiques, les vitrines deviennent cinéma et théâtre. Chaque fois que nous traversons une rue, les pare-brise des autos nous renvoient un spectacle chatoyant d'images, une sorte de collage virtuel où convergent, grâce à une série de distorsions de perspective et d'asymétries scalaires, reflets de l'extérieur et vues d'intérieur, lumières et ombres, images figées et images en mouvement.

Le thème des transparences et des reflets, qui s'impose grâce à l'introduction du verre en architecture, n'est pas récent; il naît avec le Bauhaus de Gropius, les centres commerciaux Schoken de Mendelssohn, les magasins Bata et les gratteciel de Mies van der Rohe; il s'affirme ultérieurement au cours des années 1960 avec les expériences architectoniques des centres directionnels et des commerces de l'Europe du Nord, expériences caractérisées par une radicale simplification des formes. Le productivisme de cette période tend vers l'homologation des langages et donne lieu à l'architecture sans qualité du style international. Avec le passage du Moderne au contemporain, la transparence change son langage et ses matériaux: la transparence froide du Moderne est remplacée par la transparence chaude du contemporain; comparée à la transparence algide des gratteciel miesiens et aux lames vitreuses du style international, la transparence du contemporain comporte des traits plus empathiques et plus attrayants.

La transparence froide du Moderne est celle décrite par Edward Hopper, le chantre par excellence de la solitude urbaine: dans ses figurations, qui se meuvent généralement de l'extérieur vers l'intérieur, le verre public des fenêtres et des vitrines donne accès à des intimités privées, en en dévoilant toute la fragilité humaine. À l'intérieur de la ville transparente, tout individu s'expose lui-même, n'étant plus protégé par des parois domestiques compactes et rassurantes. Dans Topâzu (Tokyo Decadence, 1992), le célèbre film de Ryu Murakami, les vitraux des hôtels qui donnent sur le panorama nocturne et crépusculaire de la Ginza mettent à nu le vécu (la vie, les vicissitudes) de la protagoniste: une prostituée japonaise qui offre ses services dans l'espace aseptique irréel des chambres des hôtels luxueux de Tokyo.

À la transparence froide du style international (et de ses avatars) s'oppose la transparence chaude des vitrines urbaines de la contemporanéité, comme celle 
peinte dans Odalisque Express (1992-1993) par Tom Blackwell (artiste de pointe de l'hyperréalisme américain). Le tableau représente la vitrine d'un magasin de vêtements où se superposent, comme sur une pellicule cinématographique, les diaphanéités de l'intérieur, avec ses chaises, mannequins et vêtements, et les reflets flous de la rue: piétons, autos, néons et l'enseigne inversée de l'hôtel Alexander's. Le fondu enchainé des figures qui reflètent les sons et les bruits de la ville imprime à la composition le rythme dynamique d'une jam session. Au centre du tableau chatoie la reproduction de la Grande Odalisque (1814) de JeanAuguste-Dominique Ingres, reflet probable d'une maxi affiche urbaine ou d'un poster exposé à l'intérieur du magasin. Composé comme un triptyque, Odalisque Express est ponctué verticalement par les châssis de la vitrine dont les signes géométriques forment le contrepoint du nu sinueux d'Ingres.

Cette diversité de transparences et de reflets de la contemporanéité, par rapport aux codes du passé (diversité mise en relief par les représentations des vitrines urbaines de Tom Blackwell), relève de deux facteurs contingents: l'un de nature technique, l'autre de nature culturelle. Le thème du verre dans la contemporanéité s'éloigne de la transparence froide typique du Moderne, de la transparence que Colin Rowe, dans The Mathematics of the Ideal Villa and Other Essays ${ }^{3}$, appelle «transparence littérale» et qui se décline en une large palette de produits: verres sablés, acidifiés, multimédiaux, interactifs, comme ceux qui ont été testés récemment par Saint-Gobain. À ces matériaux s'ajoutent les dispositifs multimédiaux, souvent appliqués au mur-rideau des façades, comme le pixel, le led, le videowall et le sunlight. La transparence est réalisée, en outre, à l'aide de panneaux micro-percés, de réseaux soudés électroniquement, et de mailles métalliques où les perforations et les vides rendent perméables des matériaux originairement opaques. L'autre aspect est lié à la culture et aux pratiques du numérique: les palimpsestes de figures concrètes et d'images reflétées, mis en valeur dans les vitrines urbaines, sont interprétés aujourd'hui de concert avec les figurations de l'électronique, grâce à leur capacité d'hybrider le réel et le virtuel et d'en déstabiliser les frontières. Les vitrines urbaines de la contemporanéité, avec leurs reflets et leurs fondus, acquièrent la même luminescence que celle des monitors. Les thèmes anticipés par Tom Blackwell se concrétisent grâce à la réalisation, par Jean Nouvel, du bâtiment de la Fondation Cartier à Paris. À travers un emploi savant de verres doubles, de cristaux acidifiés, de surfaces translucides et d'armatures vides qui auréolent le ciel, cet édifice met en scène

3. Colin Rowe, The Mathematics of the Ideal Villa and Other Essays, Cambridge (Mass.), MIT Press, 1976. 
toutes les potentialités liées, en architecture, à la superposition de transparences et de reflets.

Dans l'entretien entre Jean Baudrillard et Jean Nouvel, paru dans Les objets singuliers. Architecture et philosophie, l'architecte français dévoile les tromperies et les secrets de la Fondation Cartier, en montrant comment la superposition des transparences et des reflets déstabilise, par son déséquilibre, la tectonique de l'architecture pour la rapprocher des territoires de l'art:

Dans un bâtiment comme celui de la fondation Cartier - où je mélange volontairement image réelle et image virtuelle -, cela signifie que dans le même plan je ne sais jamais si je vois l'image virtuelle ou l'image réelle. Si je regarde la façade, comme elle est plus grande que le bâtiment, je ne sais pas si je vois le reflet du ciel ou le ciel en transparence... Si ensuite je regarde l'arbre à travers les trois plans vitrés, je ne sais jamais si je vois l'arbre en transparence, devant, derrière, ou le reflet de l'arbre. Et quand je plante deux arbres en parallèle comme par hasard par rapport à un plan vitré, je ne peux pas savoir s'il y a un deuxième arbre ou si c'est un arbre réel. Ce sont des jeux, c'est pour te mettre dans la cuisine, je dirais dans la cuisine architecturale, celle qu'on ne devrait jamais révéler et dont, de temps en temps, il faut parler. Ce sont des moyens pour l'architecte de créer un espace virtuel ou un espace mental, c'est une façon d'abuser les sens, et c'est surtout une façon de conserver un territoire de déstabilisation ${ }^{4}$.

Auparavant Colin Rowe, en comparant la «transparence littérale » du Moderne, ou encore la transparence cristalline de Taut et de la Chaîne de verre, avec la «transparence phénoménale» de la contemporanéité, avait souligné l'ambiguïté de cette dernière, considérant toutefois cette caractéristique moins comme une perte qu'une opportunité :

Thus, there is now introduced a conception of transparency quite distinct from any physical quality of substance and almost equally remote from the idea of the transparent as the perfectly clear. In fact, by this definition, the transparent ceases to be that which is perfectly clear and becomes, instead, that which is clearly ambiguous 5 .

On rencontre le thème du miroir et celui complémentaire du double à des époques différentes et dans des ouvrages disparates. Le récit gothique Strange Case of Dr Jekyll and Mr Hyde (L'étrange cas du docteur Jekyll et de M. Hyde, 1886) de Robert Louis Stevenson, The Secret Agent (L'agent secret, 1970) de Joseph

4. Jean Baudrillard et Jean Nouvel, Les objets singuliers. Architecture et philosophie, Paris, Calmann-Lévy, 2000, p. 20.

5. Colin Rowe et Robert Slutzky, «Transparency: Literal and Phenomenal » [1963], dans Rowe, 1976, p. 161. 
Conrad, Through the Looking-Glass (De l'autre côté du miroir, 1871) de Lewis Carroll, The Picture of Dorian Gray (Le portrait de Dorian Gray, 189o) d'Oscar Wilde, et le monde des miroirs du Manual de zoología fantástica (Manuel de zoologie fantastique, 1957) de Jose Luis Borges, sont autant de voix qui décrivent en littérature les effets d'étrangeté et de perte de soi causés par le thème du reflet et du double.

Le jeu complexe d'indices et de reflets inscrit à même Las Meninas (16561657) de Vélasquez, dont la trame inextricable doit être démêlée par le spectateur comme dans un roman policier, les anamorphoses des Ambassadeurs (1533) de Hans Holbein le Jeune, Le Grand Verre (1915-1923) de Marcel Duchamp, les tautologies de One and Three Mirrors (1965) de Joseph Kosuth, les portraits au miroir de Francis Bacon, le Faux miroir (1928) surréel de Magritte, les autoportraits doubles de Alighiero Boetti qui déstabilisent les frontières entre l'original et la copie, les pavillons à miroirs de Daniel Buren qui multiplient les reflets, le Serpentine Gallery Pavilion réalisé à Londres par Kazuyo Sejima et par Ryue Nishizawa comme une nuée dont le revêtement reflète l'image retournée des visiteurs, l'autoportrait de Giuseppe Penone qui, dans Renverser ses yeux (1970), remplace les pupilles par deux petits miroirs, les tableaux réfléchissants de Michelangelo Pistoletto qui capturent l'image du spectateur et qui la transforment en une partie intégrante de la composition, les reflets impossibles des flaques d'eau qui figurent dans les banlieues de Botto et Bruno constituent, dans leur ensemble, les divers langages qui nous parlent des périls de la vision et de l'art comme tromperie, en nous rappelant, à la manière de Magritte, que l'image reproduite d'une pipe «n'est pas une pipe ». Le thème du reflet traverse l'art de tous les temps. À en croire Leon Battista Alberti, le mythe de Narcisse, tel que Le Caravage l'a figuré, représente la première irruption de l'art à l'intérieur du paysage originaire.

Le thème du double en tant que figure implicite de toute composition fondée sur la symétrie est typique de l'architecture. Si la figure du reflet et celle du double débouchent, dans un contexte métahistorique, sur divers résultats, ceux-ci revêtent aujourd'hui une importance particulière qui dérive du paradigme numérique: dans la ville binaire et à l'ère de Google, les reflets sont lus en assonance avec les dispositifs de l'électronique qui déstabilisent les frontières entre réel et virtuel. Cette condition rend le problème de l'ambiguité des reflets tout à fait actuel, ainsi que l'attestent les expositions qui, ces dernières années, ont été consacrées à ce thème: de l'exposition turinoise Le miroir et son double, aménagée à la Mole Antonelliana en 1987, en passant par l'exposition française À travers le miroir, de Bonnard à Buren, organisée au Musée des Beaux-Arts de 
Rouen en 2001, jusqu'à une œuvre toute récente (Spéculation d'artiste. Quatre générations au miroir), aménagée au Musée Carlo Biliotti, dans l'Orangerie de la Villa Borghese à Rome en 2009.

\section{LA VILLE MÉdiale}

La ville médiale est enveloppée dans un filet inextricable de simulacres, d'affiches, de panneaux, tel un immense empaquetage de Christo et JeanneClaude où, pour paraphraser Adolf Loos, l'ornement n'est plus un crime. C'est la ville packaging, dans laquelle la conception traditionnelle de l'architecture, en tant que composition de formes, volumes et matériaux, est remplacée par les dispositifs immatériels de l'électronique et du numérique; à l'expressivité tectonique de la pierre, du fer et du béton succède l'insoutenable légèreté du led, du pixel, du sunlight et du videowall.

La surface des bâtiments contemporains, tour à tour translucide, opalescente et multi-médiale, représente le point de convergence de la façade libre de la modernité avec l'esthétique projective de la contemporanéité: les thèmes de plus en plus complexes de la transparence, qui n'est plus celle, cristalline, de la modernité, les diaphanéités articulées des vitrines urbaines, les procédés sérigraphiques appliqués aux surfaces vitrées et aux panneaux métalliques, les bâtiments-écrans des media buildings et les enseignes publicitaires qui enveloppent les trams, les autobus, les taxis et les bâtiments représentent ce Nuovo Cinema Paradiso (Cinéma Paradiso, 1988). Le film de Giuseppe Tornatore, dont l'histoire se déroule dans les années de l'après-guerre, décrit l'arrivée du cinéma dans un petit village de la Sicile. Ne pouvant pas accueillir tout le public à l'intérieur de la salle de projection, l'opérateur, joué par Philippe Noiret, tourne le projecteur vers l'extérieur et projette le film sur la paroi du bâtiment d'en face, créant ainsi une superposition ante litteram entre réel et virtuel, entre monde physique et monde évanescent. Le mouvement de la machine crée un effet de fondu enchaîné entre la permanence de l'existant, avec son vécu de persanes, balcons, balustrades, et l'impermanence des images en mouvement. La séquence du film de Tornatore anticipe les potentialités des nouveaux dispositifs projectifs. La ville médiale fait l'objet d'une esthétique projective qui se tourne vers la ville existante en superposant des images de synthèse aux monuments, aux sites archéologiques et aux aires géographiques afin de produire du nouveau. Cette nouvelle bidimensionnalité, qui pense la façade des bâtiments comme un support d'images en mouvement, atteste le retour à la décoration, au packaging, et met l'accent sur le thème de la peau, là où la dimension droite de la surface, 
liée à la perception des figures électroniques, s'impose sur la composition des volumes.

Les architectures qui ont recours aux dispositifs des media facades insistent sur les volumétries simples et essentielles, de façon à mettre en évidence l'œuvre des appareils numériques. Le thème de la peau des bâtiments multimédiaux représente un radical dépassement de l'idéologie fonctionnaliste du Mouvement moderne selon laquelle «la forme découle de la fonction». Selon le Mouvement moderne, la façade honnête est celle qui dévoile à l'extérieur son organisation interne et qui établit des relations de dépendance entre le plan et les perspectives. Les media buildings revendiquent, au contraire, une autonomie radicale par rapport aux prescriptions du Mouvement moderne, une autonomie poursuivie à l'aide du principe d'indépendance que Rem Koolhaas appelle «lobotomie»: la séparation chirurgicale, comparable à celle pratiquée en neurochirurgie sur les lobes frontaux du cerveau, entre extérieur et intérieur, entre front et replis secrets. Cette division par Rem Koolhaas n'est pas seulement le présupposé de toute architecture contemporaine, elle constitue aussi le principe fondamental de sa théorie fondée sur le «monumental» et sur «l'édifice générique». Dans un domaine transversal entre art et architecture, entre aménagements et performances, divers thèmes et figures donnent lieu aujourd'hui aux paysages de la ville médiale.

De nombreux architectes de renommée internationale se sont confrontés et se confrontent encore avec le virtuel. La Bibliothèque de France et le plus récent McCornick Tribune Campus Center, tous deux réalisés par Rem Koolhaas, le Media Galaxy di MVRDV, la Tour KPN Telecom de Renzo Piano, le Netherlands Institut for Sound and Vision des Neutelings figurent parmi les œuvres les plus emblématiques qui s'inscrivent dans les territoires des media buildings et des media facades. À ces ouvrages singuliers, il faut ajouter le parcours créatif de Jean Nouvel qui, dans sa longue et solide carrière, s'est engagé à sonder les multiples aspects qui lient l'architecture aux nouveaux médias, en consacrant la grande majorité de ses projets à cette « déviation » particulière. D’après Nouvel, «ce détournement-là, qui provoque la perception du sensible à faire passer non pas dans la matière mais dans l'immatériel, est une notion que l'architecture doit s'approprier ${ }^{6} »$.

Dans le Mediapark de Cologne, Nouvel interprète le thème du gratte-ciel comme un landmark multimédial, en renouvelant le legs du gratte-ciel Peugeot. À Prague, dans le centre commercial Andel, l'image agrandie de Bruno Ganz, 
l'ange métropolitain de Der Himmel über Berlin (Les ailes du désir, 1987) de Wim Wenders, surplombe la ville et la protège, tel un landmark. À Lucerne, la restructuration de l'auberge The Hotel a été réalisée en recouvrant les halls, les services, les chambres et le restaurant d'images tirées de films érotiques célèbres, créant un court-circuit entre voyeurisme et esthétique diffuse en tant qu'expression de la société contemporaine. Mais c'est surtout dans la réalisation récente à Copenhague du Concert Hall que l'architecte français fixe avec précision les principes qui président aux rapports entre architecture et nouveaux médias. Dans l'auditorium danois, la complexe fonctionnalité de l'édifice est enfermée dans une volumétrie précise: un parallélépipède net, dépourvu d'introspections, qui ne concède rien à l'organisation interne. Le caractère tranché d'un volume dénué de reliefs et de clairs-obscurs est racheté par le dynamisme des images numériques qui l'enveloppent entièrement, le dématérialisent en créant une sorte de raumplan électronique. À Copenhague, l'architecture entendue en tant que jeu savant de masses et de volumes se transforme en édifice générique qui met en évidence les ressources des nouveaux médias, conçues en tant que matériaux provenant de l'ensemble de la composition.

Dans les architectures qui ont recours aux images électroniques, on peut observer un court-circuit produit, d'une part, par la matérialité de l'édifice, c'est-à-dire par le fait que ce dernier a été situé à Paris ou à New York, et de l'autre, par la virtualité mise en scène à l'aide des images de synthèse. Si sur la façade de Times Square, on projette par exemple des images du film Gladiator (Le gladiateur, 1999) de Ridley Scott, nous sommes ici en présence d'une réalité tierce qui ne coïncide ni avec les images de New York ni avec celle de la Rome impériale. Cette réalité accrue est appelée par Paul Virilio, grâce à un heureux néologisme, "stéréoréalité », en référence à la stéréophonie qui renvoie à une profondeur acoustique accentuée par le dédoublement des sources sonores:

Aujourd'hui, nous nous retrouvons dans un monde et dans une réalité dédoublés: d'une part, il y a la contemporanéité, l'espace réel (c'est-à-dire l'espace au sein duquel nous sommes inscrits en ce moment, la perspective du quinzième siècle); d'autre part, il y a l'espace culturel, c'est-à-dire ce qui se produit à même l'interface de l'ordinateur, voire à même l'écran. Les deux dimensions cohabitent, exactement comme les hauts et les bas de la stéréophonie et de la stéréoscopie, et ce dédoublement de la réalité (c'est-à-dire l'opposition entre réalité actuelle et réalité virtuelle) engendre un effet de profondeur. De même que dans la stéréophonie on perçoit un 
effet de profondeur sonore, dans cette «stéréoréalité » on voit se dessiner un effet de relief que j’ai défini «perspective du temps réel » ${ }^{7}$.

En vertu de son statut, l'architecture déclinée dans toutes ses formes ne peut être que novatrice. Par conséquent, le corps de l'architecture est appelé à l'ère de l'électronique à une confrontation aussi laborieuse que nécessaire avec les corps de l'immatérialité: avec toutes les difficultés que la pratique de l'oxymore comporte évidemment.

La ville médiale ne coïncide pas, toutefois, de façon exclusive avec le thème des media buildings. Il y a une esthétique projective qui, à l'occasion de spectacles et d'événements culturels, à l'aide d'installations, d'événements multimédiaux et de performances, produit des hybridations significatives entre les formes du numérique et celles de la ville existante, en établissant des rapports de sens inédits entre mémoire et innovation, statique et dynamique, durée et instantanéité. Les néons de Maurizio Nannucci, les étoiles de Gilberto Zorio, les incursions romaines de Livia Cannella, la «lumière active» de Fabrizio Crisafulli, la «technologie poétique» de Fabrizio Plessi, les machines lumineuses de Yann Kersalé et de Michel Jarre, les «peintures d'architectures» de Skertzo et de Paolo Micciché constituent les voix les plus représentatives de cette light city qui superpose ses formes, impermanentes et performatives, à la stabilité des marbres et des pierres de la ville existante. La light city trouve, dans cette dialectique du dur et du malléable, la description d'une issue narrative qui se réclame des palimpsestes du passé, à travers une discontinuité programmée des langages. Bien qu'éphémères, ces actions mettent en relief des thèmes importants qui concernent le réaménagement de l'espace public et l'activation de nouvelles structures urbaines.

De nombreux artistes contemporains encouragent les greffes entre technologie informatique et art conceptuel, en poursuivant par là une esthétique projective qui unit les langages de l'art à la persistance des lieux. Jenny Holzer

7. Paul Virilio, Dal media building alla città globale: $i$ nuovi campi di azione dell'architettura e dell'urbanistica contemporanee, "Crossing» Media building, $\mathrm{n}^{\circ}{ }_{1}$, décembre 2000, p 10-11. Notre traduction de "Oggi ci troviamo in un mondo e in una realtà sdoppiati; da un lato vi è la contemporaneità, lo spazio reale (cioè lo spazio in cui ci troviamo in questo momento, la prospettiva quattrocentesca); dall'altro lato vi è lo spazio virtuale, cioè quello che accade entro l'interfaccia del computer, ovvero sullo schermo. Entrambe le dimensioni coesistono, esattamente come gli alti e i bassi della stereofonia o della stereoscopia, e tale sdoppiamento della realtà (cioè la contrapposizione tra realtà attuale e realtà virtuale) genera un effetto di campo, di profondità. Allo stesso modo in cui nella stereofonia si percepisce un effetto di profondità sonora, in questa "stereorealtà" si delinea un effetto di rilievo che ho definito "prospettiva del tempo reale”. » 


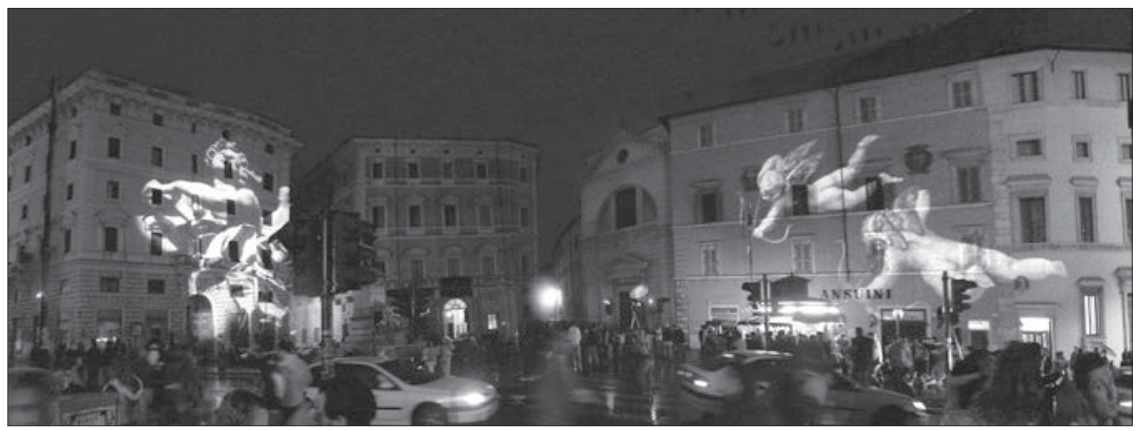

Fig 1. : Livia Cannella, Stupori in corso, Notte Bianca, Corso Vittorio Emanuele, Roma, 17 septembre 2005. Promotori: Comune di Roma, Camera di Commercio con il sostegno dell'E.T.I. e della Direzione Generale dello Spettacolo dal Vivo del Ministero per i Beni e le Attività Culturali. Coordinamento tecnico: Stefano Lattanzio-Allestimento: Artsound S.r.l.

projette des fragments de textes littéraires et des séquences de poèmes sur des cours d'eau et sur des monuments artistiques, en s'appliquant tout à la fois à corriger les éventuelles distorsions de perspective générées par le numérique. À travers ce redressement, les mots et les phrases prennent l'aspect d'une immense projection sur une plaque de verre. Tony Oursler projette, sur les façades des bâtiments, des visages qui présentent les mouvances mécaniques des premiers films muets. Les images mobiles des visages ou des torses s'accompagnent sur le plan sonore de leurs voix qui, avec une série de phrases brisées, se détendent et rient, ou bien crient et menacent, en configurant ainsi une phase prélinguistique de l'existence. Situé à l'intérieur de la tradition caricaturale qui va des polichinelles de Gianbattista et de Giandomenico Tiepolo jusqu'aux masques de James Ensor, le scénario grotesque produit par les projections de Oursler se situe à mi-chemin entre Los Caprichos (Les caprices, 1792-1799) de Goya et l'art électronique.

Mario Merz figure de premier plan de l'Arte Povera, projette la série des nombres de Fibonacci sur la Mole Antonelliana à Turin et le long des galeries de la basilique néoclassique de la place du Plébiscite à Naples. L’intervalle vide entre un chiffre et un autre représente l'espace indicible qui renvoie à l'incommensurabilité du temps et de l'espace, comme dans le paradoxe, formulé par Zénon, d'Achille et de la tortue. Enfin, à Magdebourg sur l'Elbe, ville située à sept cents kilomètres de la source du fleuve et à cinq cents kilomètres de son estuaire, Mauricio Nannucci éclaire les arcades d'un pont ferroviaire désaffecté par deux messages de couleur différente: rouge pour le message lumineux projeté sur le côté du pont tourné vers l'embouchure (constitué par l'énoncé: «Così lontano da qui [Si loin d'ici]»); bleu pour le message projeté vers la source («Da qui ancora più lontano [D’ici encore plus loin]»). 


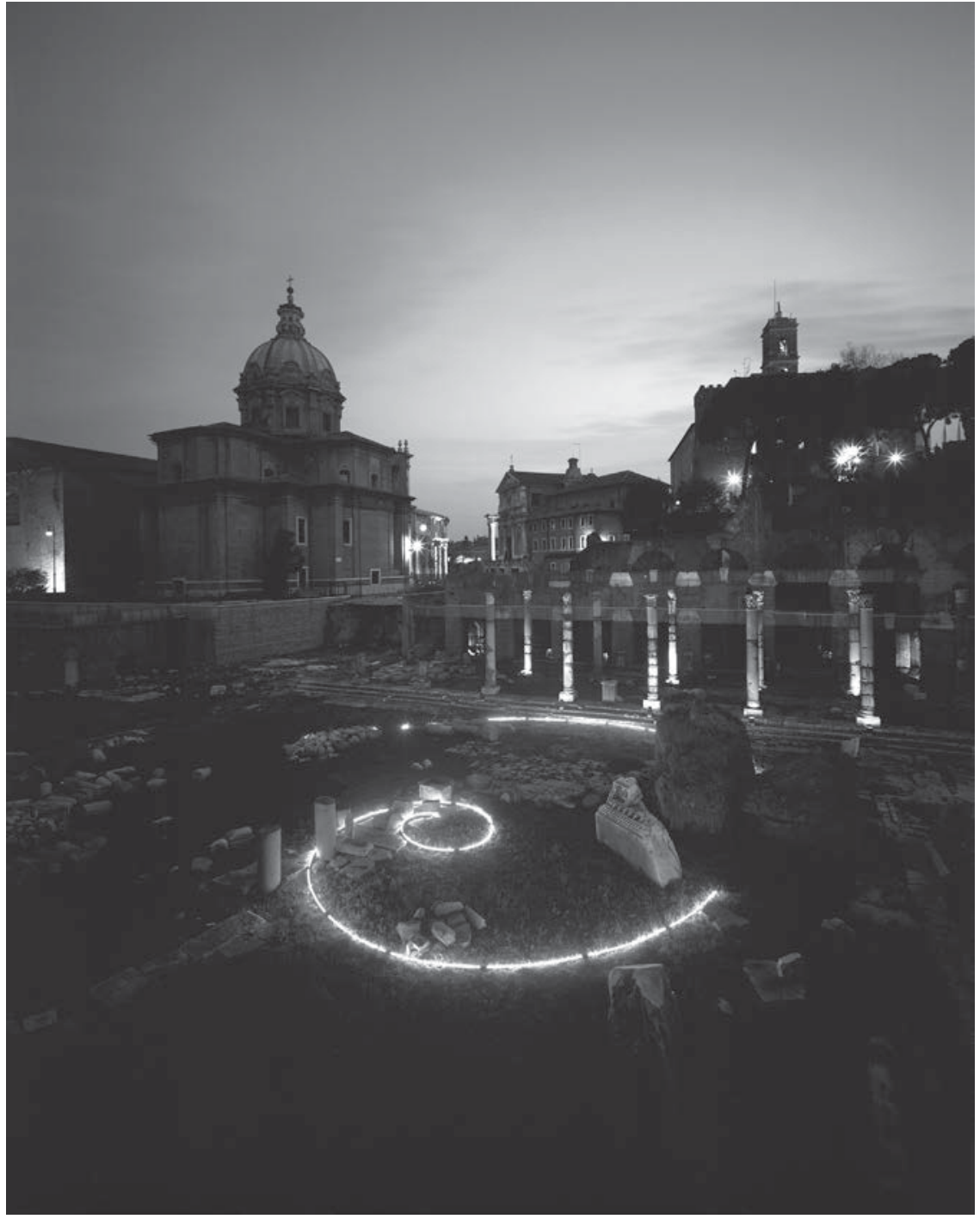

Fig. 2. : Mario Merz, Un segno nel foro di Cesare, 2003 (Photo de Claudio Abate). 


\section{LA VILLE ÉVANESCENTE}

Le paradigme électronique détermine le primat du perceptif sur le visuel, de l'immatériel sur le corporel et du système de relations sur le système des objets. La culture numérique marque, en outre, le passage de l'opaque au transparent, de l'espace au temps, de la suprématie lecorbusienne des «volumes rassemblés par la lumière du soleil» à la nature invisible des transmissions à distance et au vide de la vitesse des informations, en déterminant ce que Virilio appelle «la crise des dimensions physiques» et, par voie de conséquence, «la crise du déterminisme».

Dans la ville évanescente, on assiste à une progressive évaporation du réel. Les salles de cinéma sont menacées par YouTube, par les cellulaires et par les vidéos sur commande; les formes traditionnelles de commerce subissent la concurrence de l'e-commerce. La publicité migre de plus en plus des journaux vers Internet; les encyclopédies encombrantes de jadis se dissolvent dans l'open source de Wikipedia, alors que les livres et les quotidiens tendent à se transférer dans les e-book de Kindle; cartes routières et guides touristiques sont remplacés, à leur tour, par le navigateur satellitaire et par les technologies de localisation, comme celles préfigurées dans le film Minority Report (2002) de Steven Spielberg: à l'intérieur des magasins Gap, une affiche publicitaire s'adresse directement au protagoniste du film après l'avoir identifié à l'aide d'une lecture biométrique des yeux.

Les thèmes du numérique et du virtuel déterminent ce que Virilio appelle l'«esthétique de la disparition », phénomène qu'il faut entendre moins comme une perte que comme une singularité de la condition contemporaine. Cette prédominance du virtuel assume un rôle de guide semblable à celui exercé, dans le Moderne, par le monde des machines. Sous cet angle, le thème de la dématérialisation représente un élément significatif de confrontation entre Moderne et contemporain. Une portion consistante des écritures du Moderne apparaît comme une recherche du vide et une approximation du quasi-néant, du célèbre «beinahe nichts » de Mies van der Rohe, qui procède par creusage et par soustraction plutôt que par ajouts successifs. Dans ce secteur, nombreuses sont les contributions de l'art et les influences exercées par ce dernier sur l'architecture. Le carré blanc sur fond blanc (1918) de Malevitch, les coupes de Fontana, le monochromatisme de Klein, les soustractions de Matta-Clark, les taxinomies conceptuelles de Kosuth, les fêlures et collotex de Burri, les érosions de Pomodoro, le minimalisme lumineux de Flavin, les décollages de Rotella et les creusements de Heizer dans le désert sont autant de narrations parallèles caractérisées par la commune présence de l'absence et par la mystique du néant. 
Si l'art autrefois était matière et couleur, toile et marbre, la dimension conceptuelle fait appel aujourd'hui au vide et à l'absence, termes qui trouvent des confirmations ultérieures dans l'ère du digital.

Le drame du Moderne consiste toutefois en ce que cette recherche de la limite et sa tension vers l'a-corporel se poursuit, en ayant recours à la matérialité des instruments et des matériaux (pierre, toile, sacs, plastiques et plâtre), pour ensuite les soumettre à une pratique ultérieure de déconstruction. En configurant un réel paysage de la disparition, les nouveaux médias tâchent ainsi de résoudre les contradictions complexes de la modernité, en prenant comme point de départ l'idée de l'immatérialité qui ne suppose plus la présence d'un corps plein à soumettre à des procédés de soustraction et de dématérialisation. Le congé de la matière préparé par les nouveaux médias est représenté, dans l'art comme dans l'architecture, à l'aide de formes et de matériaux faibles et instables, comme par exemple l'eau, la lumière, la fumée, les nuages et les vapeurs: matériaux qui, associés aux technologies numériques, constituent des figures récurrentes de la ville évanescente par leur syntonie avec les districts immatériaux de l'électronique.

Le Danois Olafur Eliasson, avec son Weather Project (2003), envahit le vide de la Tate Modern avec des fumées et des vapeurs qui, nuancées par des lumières d'intensité différente, évoquent les aubes et les couchers de soleil, les variations climatiques et atmosphériques, en mettant en scène l'immatériel par excellence: le temps et son écoulement invisible. La lumière, en se réfractant dans les nuages et les vapeurs, ramène le corps dense et ferreux de la Tate Gallery à un état gazeux évanescent, à l'intérieur duquel rôde la présence dépaysée du public. Le thème de l'étrangeté devient, chez Eliasson, un instrument de connaissance. Un mélange de lumière et de buée est utilisé par l'artiste Tony Oursler qui, dans The Influence Machine (2000-2002), projette sur un nuage gazeux ses célèbres visages parlants, en accentuant le dramatique de l'expression par cet expédient scénographique. Un procédé analogue, supporté par quatre-vingt-huit phares au xénon, a été adopté après le onze septembre par le designer lumière Paul Marantz. Exécuté par une entreprise italienne, la Space Cannon vH de Fubine, il consiste en la reconstruction lumineuse des tours jumelles du World Trade Center, en interfaçant le tragique de l'évènement avec la transparence de deux faisceaux lumineux qui courent parallèlement jusqu'à six kilomètres de hauteur. À partir des années 1970, après avoir acquis une renommée internationale à Osaka grâce au Pavillon Pepsi composé essentiellement de vapeur, l'artiste japonaise Fujiko Nakaya, qui se définit comme une «artiste du brouillard ", réalise de son côté des 
sculptures de fumée et des installations urbaines de brouillard, en condensant l'eau à haute pression.

Grâce à une savante superposition d'art, de performance et d'architecture, au cours de l'Expo.02, dans la région suisse des Trois-Lacs, Diller \& Scofido combinent des arteplages plus traditionnelles, projetées pour l'occasion par Jean Nouvel, la Coop Himmelb(l)au et le groupe Multipack, avec un hybride architectonique suggestif, le Blur Building: une machine de boyaux contreventés, étendue mais transparente, légèrement posée sur les eaux du lac de Neuchâtel et accessible grâce à une plateforme étroite en fibre de verre. Les formes de cette machine rappellent les voûtes géodésiques de Buckminster Fuller, aux structures spatiales du situationniste Constant et aux machines, zoomorphiques et automouvantes, des Archigram. Le Blur Building, figure récurrente du panorama de l'architecture contemporaine, est quasiment invisible ; toutefois, il prend consistance lorsque l'eau, vaporisée par trente-trois mille saturateurs rangés le long des structures tubulaires, crée une gaine évanescente (mise en évidence pendant la nuit par la lumière artificielle), dont l'agencement change en fonction de la direction et de l'intensité du vent provenant des montagnes environnantes. Dans les vidéos électroniques de Bill Viola, l'eau est représentée alternativement comme l'alfa et l'oméga de l'existence: des corps immobiles flottent au milieu d'eaux qui présentent les traits du liquide amniotique, comme dans The Fall into Paradise (2005), alors que dans d'autres vidéos, comme The Crossing (1996), l'eau, alternant avec le feu, évoque un pouvoir destructeur en mesure d'anéantir les corps et les mémoires.

Ce flou réalisé en associant des matériaux instables tels que la lumière, l'eau et la vapeur aux technologies numériques crée une structure antipolaire par rapport aux certitudes dogmatiques du Moderne, qui soit en mesure de saisir la fluidité de la condition contemporaine que Zygmunt Bauman appelle la «modernité liquide ». Le thème du blurring est un aspect récurrent des paysages électroniques, analysé ponctuellement par Toyo Ito, dans son étude Blurring Architecture (1999), et par Peter Eisenman dans Blurred Zones (2003).

\section{LA VILLE RELATIONNELLE}

L'espace urbain contemporain est un espace multitasking qui agit comme un incubateur pour divers événements liés à l'art, au spectacle et à l'information. Le théâtre et l'art de rue, les lumières d'artiste, les jardins d'auteur, les ristotrams et les trams de l'art, les archéobus, les repas collectifs préparés dans les espaces ouverts de la ville et dans les zones vertes, avec les tables rapprochées comme 
dans une œuvre de Land Art, créent des interférences urbaines entre les lieux urbains et leurs utilisateurs. À cette utilisation de la ville, s'ajoute la contribution des pratiques interactives introduite par l'électronique: les relations de réseau, les communications $\mathrm{WiFi}$, les techniques virales d'information et les techniques de localisation qui, à l'aide de cellulaires et d'audiomaps, donnent des informations en temps réel sur les lieux que l'on visite, tout en représentant les territoires d'une nouvelle géographie électronique.

À travers le médium de l'écran tactile (pratiques interactives des surfaces sensibles et matérielles qui réagissent au toucher, aux variations de l'environnement et à celles du climat), la ville prend la forme d'un hypertexte. Dans les magasins Prada projetés par Rem Koolhaas, les miroirs technologiques intégrés aux salles d'essayage peuvent reconnaître aussi bien les vêtements, grâce à la lecture de leurs codes, que la taille et les caractéristiques du client, par la lecture de son badge. En mettant en relation leurs données, les magasins Prada peuvent orienter les choix d'achat des clients.

La ville relationnelle est la ville qui transforme l'espace urbain en un lieu d'interférences perceptives générées par le paradigme électronique. Dans la ville relationnelle, le flâneur de la contemporanéité, ayant abandonné définitivement les passages et les boulevards de Walter Benjamin, apprend, à l'aide de son iPhone, l'art de se perdre dans la forêt du réseau. Le thème de l'interactivité est un thème enraciné dans la contemporanéité. Si l'esthétique a toujours été interactive, en tant que produit finalisé à susciter des émotions, cet aspect devient particulièrement explicite dans la contemporanéité en vertu de ses implications, tant instrumentales que symboliques, dans le domaine artistique et architectonique.

Dès ses commencements, Studio Azzurro s'est engagé à configurer des espaces d'immersion où la présence de l'observateur, saisie grâce à des détecteurs auditifs, volumétriques ou tactiles, active le mouvement des projections vidéo. Les environnements perceptifs de Studio Azzurro déplacent l'attention de l'œuvre en elle-même, liée au contenu de ses projections, à la représentation des relations qu'elle instaure avec le public: le mouvement des corps dans l'espace aménagé, la pression exercée sur le plancher de la salle, un coup de toux ou le simple contact d'une plume qui effleure un touch screen peuvent déterminer la séquence et la sélection des images projetées. Ces liens subtils et imprévisibles, qui s'établissent entre les actions faibles du public et les images vidéo, constituent l'aspect central de la poétique de Studio Azzurro, axée sur la représentation de l'immatérialité des pratiques interactives en tant qu'«art léger». 
Dans Open Burble (2006), Usman Haque gonfle une série de ballons pour ensuite les libérer dans l'atmosphère. Des dispositifs spéciaux intégrés aux ballons permettent au public d'interagir avec ces derniers via leurs cellulaires. En composant des numéros de téléphone préétablis, on peut écouter les sons et les bruits produits par l'assistance mais seulement perçus d'en haut. En fonction des appels effectués, les ballons changent de couleur et rendent manifeste ce qui est d'habitude invisible, c'est-à-dire la variation du champ électromagnétique. Avec Under Scan (2005), Rafael Lozano-Hemmer réalise une performance compliquée qui suppose la participation, non consciente, du public. Grâce à des dispositifs numériques complexes, l'ombre des passants qui traversent la place principale de Leicester, en Angleterre, est remplacée par des images projectives provenant d'autres personnes. Une fois passé l'effet de surprise, ces images transmigrent ailleurs et colonisent l'ombre d'autres sujets. À Rotterdam, dans Body Movies (2001), Lozano-Hemmer projette, sur les parois d'un multiplex de la place construite par West 8, les ombres fausses de passants en les échangeant avec les images préenregistrées d'autres passants.

Lorsqu'ils sont appliqués à l'architecture, ces dispositifs activent de nouvelles relations contextuelles, comme celles mises en évidence par Toyo Ito dans la Tour des vents (1986) de Yokohama. Entre art et aménagement urbain, cette structure, réalisée à partir d'un réservoir d'eau désaffecté, enregistrait la variation des conditions environnementales telles que la pollution, les bruits et la circulation, à l'aide du changement de lumière, des reflets et des transparences: elle prenait tout à tour l'aspect d'un objet transparent ou celui d'un volume opaque et imperméable. Bien qu’elle ait été démolie dans les années 1990, cette architecture de la lumière conserve son actualité.

Les quatre villes mentionnées - opalescente, multimédiale, évanescente et relationnelle - convergent vers une lightness commune. La ville stratifiée, des fondus enchaînés, avec ses lumières et transparences électroniques, représente un affranchissement par rapport aux cartes et aux narrations de la modernité; parallèlement, elle constitue la revendication d'une condition soft opposée à la condition hard du passé. Elle scelle aussi le passage de la civilisation industrielle à la civilisation digitale, du hightech au slowtech, du visible à l'immatériel, de l'espace au temps. Lightness, donc, en tant qu'épiphanie d'un conflit difficile et dangereux entre eidos et logos qui constitue, toutefois, l'un des paradigmes les plus significatifs de la condition présente.

Vus d'en haut d'un point de vue orbital, les lumières d'artiste, les fibres optiques, les communications wireless, les techniques de localisation des vidéoguides, les pratiques interactives et les matériaux sensibles constituent les sillages 
lumineux de la nouvelle cosmologie urbaine. Une communication largement horizontale et diffuse remplace la verticalité héroïque des enseignes de la modernité. Les lumières de la Reklame Architektur et de l'effet Edison cèdent la place à l'Éden électronique de la contemporanéité, avec les paysages impalpables et évanescents de la light city.

Traduit de l'italien par Filippo Palumbo

Révision de Gilles Dupuis 